\title{
Germanica
}

\section{Progressiste ou conservateur : les paradoxes de Schoenberg}

Progressiv oder konservativ? Schönberg und seine Widersprüche

\section{Christian Merlin}

\section{CpenEdition}

\section{Journals}

Édition électronique

URL : http://journals.openedition.org/germanica/1838

DOI : $10.4000 /$ germanica. 1838

ISSN : 2107-0784

\section{Éditeur}

Université de Lille

\section{Édition imprimée}

Date de publication : 31 décembre 2003

Pagination : 201-210

ISBN : 9782913857124

ISSN : 0984-2632

\section{Référence électronique}

Christian Merlin, «Progressiste ou conservateur : les paradoxes de Schoenberg », Germanica [En ligne], 33 | 2003, mis en ligne le 03 décembre 2012, consulté le 06 octobre 2020. URL : http:// journals.openedition.org/germanica/1838; DOI : https://doi.org/10.4000/germanica.1838

Ce document a été généré automatiquement le 6 octobre 2020.

(ㄷ) Tous droits réservés 


\title{
Progressiste ou conservateur : les paradoxes de Schoenberg
}

\author{
Progressiv oder konservativ? Schönberg und seine Widersprüche
}

\author{
Christian Merlin
}

1 Musicien et penseur, Arnold Schoenberg a posé avec plus d'acuité qu'aucun autre compositeur $\mathrm{du} \mathrm{xx}^{\mathrm{e}}$ siècle les termes d'un débat esthétique qui allait dominer les décennies à venir, jusqu'à nos jours. Il s'agit, ni plus ni moins, de se confronter à la question du progrès en art et du rapport au passé, et à celle du statut de l'œuvre d'art, prise entre mouvement historique et autonomie sui generis, entre nécessité et contingence. Très vite, l'École de Vienne a été instrumentalisée dans une querelle des anciens et des modernes telle que l'histoire de la musique n'aura fait qu'en connaître sans guère de trêve. Dans une Vienne rendue frileuse par la conscience du déclin de l'Empire, il était aisé de faire de Schoenberg et de ses disciples le pendant de la Sécession en peinture, et de la Jeune Vienne en littérature. Plus tard, dans la publication de Kandinsky, Thomas von Hartmann exprimera les choses encore plus crûment, en annonçant avec triomphalisme le règne de "l'anarchie » en musique ${ }^{1}$. Il y a dans cette politisation des enjeux esthétiques un malentendu fondamental, contre lequel Schoenberg ne fut pas le dernier à s'insurger :

On a maintenant pris l'habitude de discuter des problèmes esthétiques et artistiques en termes empruntés au jargon de la politique. Des œuvres qui marquent avec modération une certaine évolution en art, en littérature ou même en musique, se voient ainsi qualifiées de «révolutionnaires» ou de "gauchistes", alors qu'elles suggèrent seulement des possibilités de progrès en matière artistique. En revanche, des œuvres conçues à la manière ancienne sont qualifiées de "réactionnaires", sans que soit pour autant expliqué ce que ce mot veut dire par contraste. [...] Ceci est totalement controuvé2.

2 La méprise est d'autant plus profonde que Schoenberg, beaucoup plus proche en pensée de Karl Kraus que des "Jeunes Viennois", est, tout comme son modèle, volontiers conservateur. Monarchiste, il aurait pu signer cette boutade bien connue de Kraus : « Ils ont cru que j'étais révolutionnaire et ils n'ont même pas su qu'en politique, je n'en étais même pas à la Révolution française ». Seul l'antisémitisme galopant ouvrira les yeux de 
Schoenberg sur les dangers de cet apolitisme à la Thomas Mann première manière. L'ambiguïté, en revanche, ne sera jamais levée chez son élève Webern, qui illustre de manière exemplaire les contradictions inextricables qui peuvent naître de la confusion entre l'esthétique et la politique. De même que Schoenberg, dans une déclaration restée célèbre, avait attribué à l'invention de la musique sérielle la faculté d'assurer la suprématie de la musique allemande pour les cent ans à venir, Webern voyait dans la création des musiciens de l'École de Vienne la possibilité de s'émanciper de l'horizon étroit et mesquin de la petite Autriche, pour reprendre enfin le train historique de la "grande Allemagne ». D'où son allégeance, inexplicable sinon, au régime nazi, et bien entendu son incompréhension face à l'hostilité dont celui-ci fit preuve à l'égard de sa musique! La même incompréhension qui était celle de Schoenberg lorsqu'il se voyait qualifié de révolutionnaire, lui qui n'imaginait pas l'art autrement qu'ancré dans l'héritage des anciens : «On se rend rarement compte qu'il y a nécessairement un lien entre l'écriture des anciens et celle des novateurs, qu'aucune technique nouvelle en art ne peut être créée qui n'ait trouvé ses racines dans le passé3. » De fait, que ce soit à Vienne ou à Berlin, Schoenberg professeur n'aura de cesse d'enseigner à ses élèves les règles canoniques du contrepoint et de l'harmonie. Quant à son Traité d'harmonie (Harmonielehre), les exemples musicaux qui l'illustrent sont très majoritairement empruntés à Bach, Mozart et Beethoven.

3 C'est encore plus frappant si l'on considère certaines œuvres clés empruntées à diverses périodes créatrices du compositeur. Emblématique de sa première époque, Verklärte Nacht (Nuit transfigurée) fut considéré par la critique comme un «veau à six têtes ", alors qu'à nos oreilles, elle semble surtout recueillir le double héritage brahmsien et wagnérien jusqu'alors considéré comme inconciliable. L'effectif du sextuor à cordes est résolument brahmsien, tandis que la tension harmonique provoquée par le chromatisme est encore proche de Tristan et Isolde, un ouvrage que Berg citera littéralement dans sa Lyrische Suite (Suite lyrique). Représentative des premières œuvres qui conduisent à la rupture avec la tonalité, la Kammersymphonie (Symphonie de chambre) op.9, par son effectif réduit et son découpage en quatre mouvements intégrés au sein d'une forme en une seule coulée, est une référence au modèle haydnien. La thématique proche des personnage de la comédie italienne, et la composition instrumentale du Pierrot lunaire, point culminant de la période atonale berlinoise, ne sont pas sans évoquer un retour au baroque. Quant aux Variationen für Orchester (Variations pour orchestre) op.31, qui apparaissent comme l'accomplissement de la méthode sérielle dodécaphonique, elles reposent sur une cellule musicale qui n'est autre que le cryptogramme $\mathrm{BACH}$. Une révérence qui n'est guère surprenante, tant il est vrai que la composition à douze sons est non seulement, pour Schoenberg, la tentative de proposer une grammaire aussi rigoureuse que la syntaxe tonale, mais elle est surtout, deux siècles après l'établissement par Bach des règles de l'échelle tempérée, une nouvelle réponse à une même question: comment équilibrer la hiérarchie entre les douze notes de la gamme chromatique?

4 Cette impression d'arbres dont l'apparence serait diverse mais les racines communes, est à l'origine de la distinction fondamentale opérée par Schoenberg entre le style et l'idée. Il y aurait ainsi deux niveaux différents dans l'œuvre d'art : le niveau visible, qui est en surface, et le niveau, pas toujours accessible au premier abord, de sa nécessité intérieure. Souvent, l'auditeur ou le spectateur se laisse abuser par l'apparence, qui relève de la contingence, et conclut à la nouveauté radicale d'une œuvre, sans voir ce 
qui la relie à ses antécédents : "Lorsqu'un changement de style survient en matière d'art, on a généralement tendance à mettre exagérément l'accent sur ce qui sépare le nouveau style de l'ancien $»^{4}$. La conclusion que Schoenberg tire de ce constat n'est pas entièrement négative, au contraire. Les manifestations extérieures de l'œuvre d'art n'étant que superficielles, l'auditeur pourra plus facilement s'y adapter. Aussi le compositeur croit-il - ou feint-il de croire - que les audaces d'écriture d'abord rejetées comme révolutionnaires ou anarchistes seront bien vite intégrées et acceptées : «On peut affirmer que l'émancipation de la dissonance est aujourd'hui chose faite et que, dans le futur, nul ne rejettera plus la musique avec douze sons pour cause d'agrégats dissonants 5 ." Aussi Schoenberg est-il le dernier à se vanter de recourir à un style moderne, car la plupart des procédés ressentis chez lui comme des hardiesses étaient déjà monnaie courante depuis le dernier tiers $\mathrm{du} \mathrm{xIX}^{\mathrm{e}}$ siècle, et font donc désormais partie de la norme : «Les irrégularités font désormais partie de la grammaire et de la syntaxe d'à peu près toutes les structures musicales. En conséquence, il n'y a plus lieu de les signaler comme des éléments d'un mérite particulier dans une composition ${ }^{6}$.»

On touche ici un nœud de la contradiction apparente qui existe chez Schoenberg entre tradition et innovation. La transmission de la nouveauté et son assimilation se feront d'autant plus facilement que l'on emploiera des formes traditionnelles. Ainsi, au moment même où le musicien met au point la méthode dodécaphonique et fustige le néo-classicisme de Stravinsky, il choisit des titres comme Suite ou Sérénade, hérités de l'époque pré-classique, et intitule les mouvements de ses op.25 et 29 "prélude", « gavotte ", « musette ", « intermezzo », « menuet », « gigue ». L'explication qu'il donne n'est pas à négliger : il s'agit de «s'efforcer de faire entrer peu à peu dans les formes anciennes un matériau sonore nouveau, plus riche en ressource $»^{7}$. Ainsi, de même qu'il existe une distinction fondamentale entre le style et l'idée, il y en aurait une, tout aussi pertinente, entre forme et matériau, qui nous permettrait de mieux penser la dialectique entre progrès et mémoire au niveau musical. Aujourd'hui encore, les meilleurs outils notionnels pour théoriser ce subtil rapport sont ceux que fournit Adorno dans sa Philosophie de la nouvelle musique (Philosophie der neuen Musik). Ce dernier soutient en effet que la matériau musical possède un caractère historique et que les procédures techniques servant à le mettre en œuvre répondent à une logique historique. En fin de compte, ce qui permet à Adorno de défendre la modernité de Schoenberg contre celle de Stravinsky, c'est que le compositeur autrichien, contrairement à son rival russe, ne s'est pas contenté de recycler des formes anciennes, mais a permis au matériau même de réaliser des progrès notables.

6 Dans cette conception esthétique, le progrès s'effectue en quelque sorte par sédimentation violente, qui définit une véritable théorie du vestige :

Toute œuvre importante laisse des traces dans son matériau et dans sa technique ; suivre ces traces définit l'art moderne comme « ce qui est à faire ", non pas comme flair d'une mode qui se prépare. [...] Les traces abandonnées dans le matériau et dans les procédures techniques auxquelles adhère toute œuvre d'art qualitativement nouvelle sont les cicatrices, les points où les œuvres précédentes ont échoué. Tout en souffrant de ces cicatrices, l'œuvre nouvelle se retourne contre celles qui ont laissé de traces ${ }^{8}$.

C'est ainsi qu'Adorno traite l'aporie du progrès en art : la nouveauté n'y possède pas de vertu émancipatrice, ce qui s'oppose à l'idée schoenbergienne selon laquelle la musique dite atonale n'est en réalité que «l'émancipation de la dissonance». Cette logique d'affranchissement d'une servitude - pour la dissonance : être nécessairement en 
attente de sa résolution - ne concorde pas tout à fait avec la pensée adornienne des entailles qui se recouvrent à force de lutte. Cependant, si Adorno manifeste toujours son attachement à la période de libre atonalité de Schoenberg, car son côté informel a incontestablement fait avancer le matériau, il exprima toute sa réticence à l'égard de la méthode dodécaphonique, car sa prétention à l'universalité intemporelle est pour lui une manière de refuser l'historicité de l'œuvre d'art. Il ne saurait y avoir de nouveauté absolue, et en bon dialecticien, Adorno fait de la présence d'éléments régressifs dans les musiques de Mahler de Berg précisément le facteur même de leur modernité. Voilà au moins un point commun évident entre Adorno et Schoenberg: une certaine forme d'anti-avant-gardisme. "Je dois avouer que je suis de ceux qui n'attachent guère de prix à l'originalité $»^{9}$, déclara sans ambage le compositeur, qui était du reste tout prêt à reconnaître le caractère daté de toute création artistique : « De ce qu'un changement de style en art n'implique pas nécessairement un progrès, on peut prévoir qu'il sera extrêmement difficile d'établir des critères de jugement conservant leur valeur à travers toutes les époques ${ }^{10}$.»

8 Adorno est toujours soucieux de sauver au moins l'apparence de l'œuvre d'art comme totalité close et organique, soumise à une causalité temporelle. C'est pourquoi ses attaques ne se dirigent pas seulement contre le Stravinsky néo-classique - cible favorite de Schoenberg -, mais aussi contre le jeune Stravinsky, trop proche à son goût du dadaïsme et du surréalisme. Adorno reproche à ce dernier la désorganisation du matériau, l'émancipation de l'effet, désormais gratuit puisqu'il n'est plus intégré au tout. Il en résulte un primitivisme qui est régression plus que progrès, et qui est précisément ce dont Adorno fait grief aux premières pièces pour piano de Schoenberg: "L'origine de l'atonalité comme affranchissement complet de la musique des conventions, a précisément en cela quelque chose de barbare. [...] Le progrès lui-même, dans son refus de la convention, a quelque chose d'enfantin, de régressif. Les premières compositions atonales de Schoenberg, en particulier ses pièces pour piano op.11, effrayaient plus par leur primitivisme que par leur complexité ${ }^{11}$. Cette question, on le sait, sera au centre du Docteur Faustus de Thomas Mann. À l'indétermination et à l'anarchie, Adorno préfère une dialectique de la désorganisation, à l'intérieur de la clôture de l'œuvre, telle qu'il la trouve par exemple à l'œuvre chez Mahler. Rien d'étonnant à ce qu'Adorno ait fait peu de cas des musiques aléatoires.

9 Face à la difficulté de penser le matériau musical en lui-même, une des apories les plus déconcertantes de la philosophie de la nouvelle musique, Schoenberg nous invite pour sa part à le considérer en fonction de sa mise en forme au sein de l'œuvre. D'ailleurs, s'il doit exister un progrès en art, c'est uniquement à ce niveau-là : « le progrès en musique est avant tout un perfectionnement des méthodes de présentation des idées ${ }^{12}$. " Ainsi, Schoenberg nous met en garde contre une approche trop historiciste du matériau, telle qu'elle a pu tenter Adorno, mais il nous invite assurément à penser historiquement les outils permettant d'exprimer ce matériau. C'est que, si Schoenberg est obsédé par la pureté de l'idée et la crainte de la voir travestie ou falsifiée dès qu'elle est exprimée, il est aussi constamment attaché à l'intelligibilité de l'idée. En cela, il est indéniablement homme des Lumières, héritier de l'Aufklärung. Schoenberg ne voit donc rien de choquant dans l'utilisation des formes héritées du passé, si celles-ci peuvent concourir à une clarification de l'énonciation du matériau. Surtout si les techniques d'autrefois sont enrichies et améliorées, à l'exemple du contrepoint baroque perfectionné par les classiques et les romantiques, au service de l'art de la variation : 
On pourrait s'étonner que des compositeurs classiques comme Haydn, Mozart, Beethoven, Schubert, Mendelssohn, Schumann, Brahms et même Wagner, qui ont succédé au sommet contrapuntique représenté par Bach, mais dont le style est essentiellement homophonique, aient si souvent introduit $\mathrm{du}$ contrepoint strict dans leurs œuvres; ce contrepoint ne différant de celui de Bach que par les progrès que le temps a apportés à la musique, autrement dit par le développement plus poussé qui assure la variation du thème ${ }^{13}$.

10 Ainsi de Brahms, utilisant pour le finale de sa Symphonie $n^{\circ} 4$ une passacaille, technique ancienne par excellence, qui plus est sur la basse d'un thème issu de Bach, mais en l'adaptant à un langage harmonique ayant considérablement évolué. Il n'est d'ailleurs pas surprenant de voir Schoenberg choisir Brahms comme porte-étendard du progressisme en musique, comme s'il y avait plusieurs âges dans l'histoire de la musique, des balbutiements de l'enfance à la maturité de l'adulte :

Mon but est de lier des idées à des idées. Peu m'importe ce que telle idée matérialise ou signifie, dans le groupe dont elle fait partie, [...] je lui demande seulement de n'avoir pu faire autrement que de se trouver là. [...] Il me semble que le progrès apporté par Brahms en musique doit inciter les compositeurs à écrire de la musique pour adultes ${ }^{14}$.

11 Dans le même esprit, ce qu'admire le plus Schoenberg chez Wagner, ce n'est pas tant un langage harmonique dans lequel il est tentant mais un peu facile de voir un précurseur de la musique atonale, qu'une souplesse inédite dans le maniement des thèmes, qui permet de les varier en échappant aux contraintes académiques du développement: «Wagner contribua de son côté au progrès des formulations constructives par sa technique de la répétition, variée ou non, du fait qu'elle le libérait de l'obligation de travailler plus longuement que nécessaire sur des élements qu'il avait déjà clairement exposés ${ }^{15}$.» Un apport dont Schoenberg ne manque pas de remarquer qu'il peut s'accompagner d'une certaine forme d'appauvrissement, surtout lorsqu'il s'agit de faire passer son message musical auprès du plus grand nombre : «Les exigences de la grande masse se firent encore plus impérieuses lorsque Wagner bouleversa la forme en liaison avec les progrès qu'apportait sa langue harmonique. [...] Wagner se contenta, pour graver ses leitmotive dans la mémoire du public, de les répéter par séquences ou demiséquences ${ }^{16}$. »

12 Ainsi, à la différence de la théorie adornienne de la trace, du vestige laissé par une cicatrisation douloureuse, c'est plus à un mouvement conjoint de gain et de perte que Schoenberg assimile le progrès en art. Tout apport nouveau s'accompagne immanquablement d'une déperdition par ailleurs, les deux finissant par se compenser. À titre d'exemple, Schoenberg met sur le compte de la densité croissante de l'expression atteinte par le langage musical l'évolution vers des formes de plus en plus brèves et ramassées, comme pour contrebalancer le risque d'enflure hyperbolique d'une musique qui pourrait ne jamais s'arrêter: "Notre instinct de la forme ne nous avait pas trompés en nous obligeant à compenser l'extrême intensité du contenu émotif par une très grande concision. Ainsi avions-nous tiré inconsciemment les conséquences d'une nouveauté qui, comme toute autre nouveauté, détruisait quelque chose en même temps qu'elle produisait quelque chose ${ }^{17}$. " Il serait par exemple difficile d'imaginer le monodrame Ewartung (Attente), oscillographe de passions violentes et obsessionnelles traduites par une musique qui ne se répète jamais, autrement que concentré en un acte ramassé. Il n'en reste pas moins que ce choix de la brièveté ne fut pas ressenti par Schoenberg uniquement comme une nécessité historique, mais aussi comme une 
contrainte douloureuse, puisqu'il n'eut de cesse d'élargir sa méthode compositionnelle à la grande forme.

13 Cette conscience aiguë d'un devenir historique de la création artistique fait de Schoenberg tout le contraire d'un apôtre de la tabula rasa. Mais il ne faudrait surtout pas en conclure que, sous prétexte qu'il reconnaît une dette envers les anciens, il se voit uniquement comme un continuateur qui met ses pas dans ceux de ses prédécesseurs. Cette idée d'une fin de l'histoire lui est totalement étrangère :

Un juge superficiel pourrait tenir la composition avec douze sons comme l'aboutissement d'une époque qui vit l'évolution du chromatisme. [...] Mais [...] je pense que la composition avec douze sons et ce que bien des gens appellent à tort la musique atonale ne marquent pas la fin d'une ère révolue, mais bien le commencement d'une ère nouvelle ${ }^{18}$.

14 C'est pourquoi Schoenberg semble ressentir plus durement les critiques qui lui sont adressées à partir des années 20 par de jeunes créateurs avant-gardistes, alors que jusqu'ici ses détracteurs se recrutaient essentiellement dans le camp des conservateurs :

Il y quinze ans, c'étaient surtout des réactionnaires et des artisans à l'esprit commercial qui contestaient la composition avec douze sons. Leurs objections [...] partaient d'une horreur pré-wagnérienne de la dissonance pour aboutir au reproche, plus romantique et plus sentimental, de " cérébralisme ». Autrement dit, mes compositions manquaient d'inspiration, c'étaient des constructions arides, de simples épures d'ingénieur. [...] Mais il se trouve qu'aujourd'hui, les adversaires de ma manière d'écrire ont converti un nombre considérable de jeunes compositeurs. [...] Ce n'est pas la première fois qu'on lance le cri de Ars Nova!: l'histoire se répète ${ }^{19}$.

Schoenberg ne s'attendait visiblement pas à cette accélération du temps, qui fait que, en quelques années, le révolutionnaire d'hier passe pour rétrograde aux yeux des nouveaux progressistes. Mais il ne s'en formalise pas outre mesure, car pour lui la nouveauté est un devoir moral et non un simple slogan publicitaire :

Que peut bien vouloir dire musique nouvelle ? [...] Il n'est pas de chef-d'œuvre qui n'ait apporté un message nouveau à l'humanité, il n'est pas de grand artiste qui se soit dérobé à ce devoir. Tel est le code de la véritable grandeur en matière d'art. Dans toutes les œuvres des grands maitres, nous relèverons toujours ce caractère de nouveauté, qu'il s'agisse de Josquin des Prés, de Bach, de Haydn ou de n'importe quel génie. Pour cette raison que Art égale Art nouveau. L'idée que le slogan Musique nouvelle! pourrait changer quelque chose au cours normal de l'évolution musicale est sans doute issue de cette croyance que l'histoire se répète ${ }^{20}$.

16 La tension entre nouveauté et tradition qui se dégage de tous les développements précédents n'est une contradiction qu'en apparence. Il convient seulement de ne pas oublier que la pensée schoenbergienne est profondément dialectique : aussi le novateur n'a-t-il de raison d'être qu'en tant qu'il devient à son tour fondateur d'une tradition. "Mon mérite est d'avoir écrit une musique véritablement nouvelle qui, de même qu'elle est issue de la tradition, est destinée à devenir une tradition ${ }^{21}$. Où l'on rejoint les termes du débat politique auxquels on avait dans un premier temps cherché à échapper. Ce que Hanns Heisler ne manque pas de relever, non sans perfidie, en évoquant son maître Schoenberg : «Il est le vrai conservateur : il donne naissance à une révolution pour pouvoir être réactionnaire ${ }^{22}$." 


\section{NOTES}

1. Almanach du Cavalier bleu (Almanach « Der blaue Reiter). Paris (Klincksieck) 1987.

2. "Est-ce loyal?", manuscrit original anglais daté du 2 décembre 1947, publié dans Music and Dance in California and in the West, Hollywood, 1978. In : Le Style et l'idée, écrits réunis par Leonard Stein, nouvelle édition présentée par Danielle Cohen-Lévinas. Paris (Buchet/Chastel) 2002, p. 191. Tous les extraits de textes de Schoenberg cités dans cet article seront tirés de ce recueil, que nous identifierons désormais par l'abréviation $S$ et $I$.

3. "Auto-Analyse ", publié en anglais dans Program Book of New Friends of Music, New York, 13 novembre 1949. In : $S$ et I, p. 61

4. "Comment j'ai évolué ", manuscrit en anglais du 2 août 1949, paru en allemand sous le titre « Rückblick» dans les éditions de septembre de Stimmen (Berlin) et Österreichische Musikzeitschrift (Vienne). In : $S$ et I, p. 71.

5. « La Composition avec douze sons II », manuscrit non daté, sans doute écrit entre 1946 et 1948. In : S et I, p. 189.

6. «Brahms, le progressiste» («Brahms, the Progressive»), paru dans Style and Idea, Belmont Music Publishers et Faber \& Faber Limited, 1950. Style and Idea est le premier recueil, en anglais, d'écrits théoriques de Schoenberg, et est de dimensions beaucoup plus réduites que celui auquel nous faisons référence dans sa traduction française. In : S et I, p. 332.

7. « Musique nouvelle» (« Neue Musik»), manuscrit de 1923. In : S et I, p. 114.

8. Theodor W. Adorno : Théorie esthétique. Paris (Klincksieck) 1989, p. 57.

9. «Critères de jugement de la musique " (« Kriterien musikalischer Werte »), conférence en allemand de 1927, version en anglais en 1946 publiée dans Style and Idea, 1950. In : S et I, p. 104.

10. Ibid., p. 105.

11. Theodor W. Adorno: Philosophie der neuen Musik, Francfort (Suhrkamp Taschenbuch) 1976, p. 46.

12. «Brahms, le progressiste », op. cit., p. 308.

13. "On revient toujours ", manuscrit en anglais daté d'octobre 1948, publié dans le New York Times du 19 décembre 1948 puis dans Style and Idea, 1950. In : S et I, p. 90.

14. «Brahms le progressiste », op. cit., p. 314.

15. Ibid., p. 342.

16. «Critères de jugement de la musique », op. cit., p. 106.

17. «La Composition avec douze sons I », publié dans Style and Idea, 1950. In : S et I, p. 165.

18. «La Musique nouvelle, la musique démodée, le style et l'idée » (« Neue und veraltete Musik, oder Stil und Gedanke »), manuscrit en allemand de 1933, puis conférence en anglais de 1933/34, révisée en 1946 et publiée dans Style and Idea, 1950. In : S et I, p. 99.

19. «Un Tournant» («Change of Direction»), publié en espagnol dans Nuestra Musica, Mexico, 1952. In : $S$ et I, p. 116.

20. « La Musique nouvelle, la musique démodée, le style et l'idée », op. cit., p. 94.

21. Ibid.

22. Musikblätter des Anbruchs, numéro spécial, 1924. 


\section{RÉSUMÉS}

Arnold Schoenberg est à l'origine d'un tournant dans l'histoire de la musique, qui passe en général pour une véritable révolution, et pourtant le compositeur éprouvait de la peine à s'imaginer dans la peau d'un révolutionnaire, puisqu'il se considérait comme l'héritier de la grande tradition européenne, celle d'un Bach ou d'un Beethoven. Notre étude se propose d'examiner le rapport complexe de Schoenberg à la notion de progrès en art. Au fond, Schoenberg est un héritier des Lumières, pour qui l'intelligibilité du propos est d'une importance extrême : si les formes, dont la fonction est de transmettre le matériau, sont d'ordre tout à fait historique, les idées musicales, elles, sont en soi anhistoriques. C'est pourquoi il n'est pas étonnant que les œuvres les plus radicales de la période dodécaphonique recourent à l'habillage de formes conventionnelles issues du baroque et du classicisme. Cette tension fait de l'esthétique de Schoenberg un va-et-vient incessant entre nouveauté et tradition, qui repose sur une relation dialectique : à ce titre, il est légitime de se demander avec Hanns Eisler si Schoenberg n'a pas provoqué une révolution pour mieux devenir à son tour le fondateur d'une tradition.

Arnold Schönberg verdankt man eine Wende in der Musikgeschichte, die oft als eine richtige Umwälzung gilt, aber dem Komponisten fiel schwer, sich selbst als Revolutionär zu empfinden, da er sich eher als Erbe der europäischen Tradition eines Bach oder Beethoven betrachtete. Anliegen dieser Studie ist es, sein komplexes Verhältnis zum künstlerischen Fortschritt zu untersuchen. Grundsätzlich ist Schönberg ein Aufklärer, der der Deutlichkeit und Verständlichkeit des Vortrags höchste Bedeutung beimisst: sind die Formen, die dazu dienen, das Material zu vermitteln, ein durchaus historisches Phänomen, so sind die musikalischen Ideen an sich unhistorisch. Deshalb kommt es nicht von ungefähr, dass Schönberg seine radikalsten Werke der Zwölftonperiode mit herkömmlichen Formen des Barock und der Klassik umhüllt hat. Diese Spannung führt Schönbergs Ästhetik zu einem ständigen Hin- und Herpendeln zwischen Neuigkeit und Tradition, dem letzten Endes eine dialektische Beziehung zu Grunde liegt: in dieser Hinsicht darf man sich mit Hanns Eisler fragen, ob Schönberg nicht eine Revolution ausgelöst hat, um selber zum Gründer einer Tradition zu werden.

\section{AUTEUR}

\section{CHRISTIAN MERLIN}

Université Charles-de-Gaulle - Lille 3 